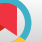

\title{
Assessing the Effect of Training the Safe Transfer Checklist on the Quality of Intrahospital Patient Transfer: An Interventional Study
}

\author{
Sadegh Akrami ${ }^{1}$, Gholam Hosein Sargazi (iD) ${ }^{2}$, Leila Safabakhsh ${ }^{3}$ and Zahra Pishkar Mofrad (iD ${ }^{4, *}$ \\ ${ }^{1}$ MSc Nursing Student, School of Nursing and Midwifery, Zahedan University of Medical Sciences, Zahedan, Iran \\ ${ }^{2}$ Anesthesia Department, Paramedical School, Zahedan University of Medical Sciences and Health Services, Zahedan, Iran \\ ${ }^{3}$ Community Nursing Research Center, Zahedan, Iran \\ ${ }^{4}$ Surgical Nursing Department, School of Nursing and Midwifery, Zahedan University of Medical Sciences, Zahedan, Iran \\ "Corresponding author: School of Nursing and Midwifery, Mashahir Square, Postal Code: 9716913396, Zahedan, Iran. Tel: +98-5433442481, Email: pishkarz@gmail.com
}

Received 2019 November 15; Accepted 2019 November 23.

\begin{abstract}
Background: Intrahospital transfer of critical patients is due to diagnostic, therapeutic objectives or hospitalization in specialized units. This transfer could prompt cardiovascular, respiratory, and physical problems for patients with critical conditions.

Objectives: The present study attempts to determine the impact of training nurses in regards to the use of safe transfer checklist on the quality of in-hospital transfer of critical patients.

Methods: This quasi-experimental study was performed in two hospitals affiliated with Zahedan University of Medical Sciences between July and December 2018. Khatam Hospital and Ali ebne Abitaleb Hospital were selected as the control and intervention (checklist) groups, respectively. In the control hospital, 65 cases of intrahospital transfer were evaluated by observing the performance of the transfer team, and the researcher completed the checklist. Next, in the intervention hospital, the researcher organized a workshop on using the safe transfer checklist. After a month, 65 cases of intrahospital transfer in this hospital were monitored and the safe transfer checklist was completed. Data were analyzed in SPSS 22 using chi-square test and independent $t$-test. P $<0.05$ was considered statistically significant.

Results: The mean age of patients was 43.68 years in the control group and 47.95 years in the intervention group. The majority of patients (76.9\% in the control group and 61.5\% in the intervention group) were male. The mean score of intrahospital transfer quality was $11.21 \pm 2.84$ in the control group and $16.52 \pm 2.08$ in the intervention group. Independent $t$-test showed a significant difference between the two groups in terms of transfer quality $(\mathrm{P}<0.001)$, with the intervention group scoring higher than the control group. Conclusions: Teaching nurses to apply the safe transfer checklist in moving critical patients inside the hospital can improve the quality of transfer, and, ultimately increase the safety of critical patients.
\end{abstract}

Keywords: Education, Checklist, Quality, Intrahospital Transfer, Critical Patient, Nurse

\section{Background}

Transferring patients from Emergency and ICU to carry out diagnostic and therapeutic examinations and procedures is one of the essential components of care (1). The decision to move a critical patient inside a hospital or a treatment center is based on weighing the potential benefits against potential risks involved $(2,3)$. Preferably, the patient should not be transferred for tests and procedures that are unlikely to change the care process (4). However, if it is unavoidable, one can mitigate possible risks by taking measures such as patient stabilization before transfer, coordination with the destination unit, establishing appropriate communication between attending staff, checking transportation and monitoring equipment, and documentation (5).
Replacing life-saving medical devices in the Emergency and ICU by portable equipment can lead to unwanted physiological changes in patients, especially those with critical conditions. Complications may also occur during transfer for uncontrollable causes outside the unit: cardiac or respiratory arrest, hypotension, hypertensive crisis, aspiration, life-threatening arrhythmias, increased intracranial pressure, heart attack, respiratory change, increased airway pressure, airway obstruction due to secretions, excessive coughing, anxiety, and bleeding. These outcomes could bring about medium or long-term effects that persist for up to four hours after transfer. They are most commonly seen in patients undergoing mechanical ventilation with positive end-expiratory pressure and continuous infusion of vasoactive medications (6). Statistics indicate that $2.5 \%$ 
to $75 \%$ of patients experience transfer-related complications (7). Therefore, it is crucial to stabilize patient's conditions prior to transfer, accurately prepare the equipment, train the staff involved, and monitor the incidents during the transfer process (8).

Nurses are essential members of the treatment team, and their role in preventing complications and achieving optimal therapeutic outcomes is undeniable; consequently, it is necessary for them to develop their nursing care knowledge in order to maintain professional standards (9). Meanwhile, studies suggest that existing educational programs on transferring and caring for critically ill patients are not adequate and effective, and neither medical nor nursing staff receive any training before transferring critical patients; at best, these instructions have been quite limited (10-12). While transferring critical patients, nurses endure a great deal of stress because of their high vulnerability (11). Cognitive performance is negatively affected by increasing fatigue and stress. Stressful situations can also lead to errors in judgment, impair the implementation of standard procedures, and prevent nurses from exercising their skills fully (12). To avoid transfer complications, existing guidelines recommend making changes to clinical practice by employing trained personnel and suitable equipment, monitoring all movements throughout the process (including diagnostic procedures), and using a checklist to ensure the correct delivery of services and resources (13). Many researchers argue that medical checklists can help prevent human error, mitigate injuries, and cut various costs $(8,14)$. The benefits of using checklists include their comprehensive nature, optimizing the usage of resources, and enhancing the performance of the treatment team. Undoubtedly, there are downsides to checklists as well, some of which concern the time they require (due to too many items), dependency on observation criteria, likelihood of bias on the part of the person who fills them, lack of attention to details, fatigue, and improper usage (15). In fact, checklist provides a standard and safe method for assessing intrahospital transfer (16). Therefore, using a checklist that summarizes the essentials before, during, and after this procedure can improve the quality of patients' intrahospital transfer (12). In the study by Farnoosh et al., implementing the patient transfer protocol in the emergency department reduced unexpected complications (17). There is evidence illustrating that patients who are transferred by an experienced treatment team encounter fewer adverse events (18). Specifically, the incidence of adverse events in the case of transferring patients by a specialized team is $15.5 \%$; however, this rate soars to $75 \%$ when transference is carried out by non-qualified people (19).

Based on the study by Choi et al., training nurses through safe transfer guidelines and intrahospital transfer checklists significantly reduces the incidence of unexpected events when transferring emergency patients within the hospital (8). Habibzadeh et al., also reported that interactive training can have a positive impact on the performance of nurses who are in charge of transferring patients (20). Furthermore, by determining the risk of transferring critically ill patients, it is possible to prevent many serious complications (21). Considering the experience of being present in therapeutic settings as nurses or patient companions, the present researchers have realized that transferring patients, especially those with critical conditions, does not currently follow safety standards. After searching national and international databases, the authors found that the subject of patient transfer has not been extensively studied, even though having sufficient knowledge of patient care during intrahospital and interhospital transfers is crucial.

\section{Objectives}

The present study was conducted to explore the effect of training the usage of safe transfer checklists on the quality of intrahospital transfer of critical patients admitted to two hospitals of Zahedan University of Medical Sciences.

\section{Methods}

After the approval of the Ethics Committee of Zahedan University of Medical Sciences (IR.ZAUMS.REC.1397.192), this quasi-experimental two-group study was collected at the Khatam Hospital Hospital and Ali ebne Abi Taleb Hospital (Zahedan) in 2018. The study population consisted of all intrahospital transfers of critical patients and all nurses responsible for this task. The samples included all nurses in charge of intrahospital transfer of critical patients who worked at the emergency, ICU, and CCU of the above-mentioned hospitals during data collection. The inclusion criteria for patients were: critical patients who were ordered by the treating physician to be transferred inside the hospital for diagnostic or therapeutic procedures, obtaining a minimum score of 14 and greater based on the risk score for interhospital transport, patient accompaniment by the ward nurse during the transfer process, and lack of patient accompaniment by the physician. The inclusion criteria for nurses were working in the CCU, ICU, or emergency for at least six months, no record of attending any patient transfer workshop in the last 6 months, and consenting to take part in the study. On the other hand, the exclusion criteria for nurses were leaving the workplace or moving from the relevant ward during the study period. 
Based on the research by Habibzadeh et al. (22), the sample size was estimated at 65 intrahospital transfers for each group (total $=130$ ) with a $95 \%$ confidence interval and $80 \%$ power.

$n=\frac{\left(Z_{1-\frac{\alpha}{2}}+Z_{1-\beta}\right)^{2}\left(S_{1}^{2}+S_{2}^{2}\right)}{\left(\bar{x}_{1}-\bar{x}_{2}\right)^{2}}=65$

$\mathrm{Z}_{1-\alpha / 2}=1.96 ; \mathrm{S}_{1}=87.9, \bar{x}_{1}=26.49, \mathrm{Z}_{1-\beta}=85.0, \mathrm{~S}_{2}=27.10$, $\bar{x}_{2}=95.54$.

Data collection tools included: a demographic questionnaire for nurses and another for patients, a profile registration form for intrahospital transfer, and a researchermade checklist for assessing the quality of intrahospital transfer. The researcher completed these forms by interviewing the nurses, examining patients' medical records and documents related to patient transfer process, and directly observing the procedures.

The transfer risk checklist includes 14 items: systolic blood pressure, mean arterial blood pressure, central temperature, heart rate, respiratory rate, pulse oximeter, hematocrit, arrhythmia, primary pacemaker, central venous access, patient's consciousness level, cardiopulmonary monitoring, respiratory support, and artificial airway (21). This scale was scored between 0 and 28 , representing the level of risks associated with transferring the patient. Patients scoring 14 to 28 were placed in the highrisk group and were enrolled in the study. The profile registration form of intrahospital transfer comprised questions about the personnel accompanying patients during the transfer process, transfer time (shift), patient's age, gender, and medical diagnosis, transfer vehicle, transfer duration, and transfer distance.

The checklist for assessing the quality of patient transfer included three parts filled in by the researcher: the first part (13 questions) dealt with performance standards prior to patient transfer; the second part (8 questions) covered performance standards during patient transfer; and the third part (5 questions) assessed performance standards after patient transfer was over. This scale featured 26 yesno statements regarding the safety standards of intrahospital transfer. For each item, nurses were respectively given a score of one (yes) and zero (no) in case of adherence and non-adherence to an individual standard of patient transfer; thus, higher scores referred to more favorable intrahospital transfers (22). In this checklist, the scores of items, which did not need to be taken into account during the transfer process (such as "the patient does not need oxygen during transfer") were generalized to other items and were marked in a column entitled "no standard for review".
Then, the sum scores of transfers were calculated and reported on average. The validity of the instrument was determined through content validity, and its reliability was confirmed based on internal consistency and Cronbach's alpha (0.75) in the study by Habibzadeh et al. (22). In the present study, reliability of this tool was investigated using inter-rater reliability, whereby ten transfer items were evaluated by two raters and reliability was established based on correlation coefficient (0.91).

After obtaining the necessary permits to enter the research environment, Khatam Hospital was selected as the control group and Ali ebne Abi Taleb Hospital was considered the intervention group (checklist training). Critical patients were determined through convenience sampling among qualified intrahospital transfers. In the control group hospital, after the informed consent was obtained from the patients (or their companion) and the nurses in charge of the transfer, the researcher observed and evaluated 65 cases of qualified intrahospital transfers based on the transfer quality checklist.

In the intervention group, after necessary coordination with the hospital educational supervisor, a two-hour workshop was held in which patient transfer instructions were presented to the nurses by lecture and PowerPoint slides. They were also taught on how to use the safe transfer checklist when moving patients inside the hospital. The educational content for safety standards of intrahospital transfer was prepared using authentic books and electronic resources. Once approved by 10 of the faculty members of the School of Nursing and Midwifery (Zahedan University of Medical Sciences) as well as nursing directors of the related hospitals, the resulting materials were used in the study. One month after the workshop, the researcher selected 65 cases of intrahospital transfer, which met the inclusion criteria. After the patients or their companions gave their informed consent, the performance of the trained nurses was observed based on the checklist and the questionnaires were completed.

Data were analyzed in SPSS 22 using descriptive statistics (frequency, percentage, mean, standard deviation), independent $t$-test (to investigate the mean of quantitative variables in the study groups), and chi-square test (to compare the qualitative variables of the two groups). $\mathrm{P}<0.05$ was considered statistically significant.

\section{Results}

Totally, 130 patients completed the study. Based, on the results, there was no significant difference in the mean and standard deviation of the patients' age in the intervention group ( $47.95 \pm 21.32$ years) and the control group (43.68 \pm 21.15 years $)(\mathrm{P}=0.89)$. The majority of patients 
in both groups were male, 50 (76.9) in the control group and 40 (61.5) in intervention group 40 (61.5). The mean age of nurses participating in the study was 30 years; $85 \%$ of the subjects were nursing experts in both groups. However, $40 \%$ of nurses in the control group were employed on contract basis, which was statistically significant from its percentage in the intervention group $(\mathrm{P}=0.02)$. The frequency distribution of other transfer-related variables is presented in Table 1.

The intervention group scored significantly better than did the control group. In this regard, the independent $t$-test revealed a significant variation between the two groups $(\mathrm{P}<0.001)$ (Table 2 ).

\section{Discussion}

The purpose of this study was to explore the impact of training the usage of checklists on the quality of intrahospital transfer of critical patients. The results of the present study confirm the findings of other researchers who suggest that one can substantially reduce the risk of moving critical patients inside the hospital provided that appropriate measures are taken before and during the transfer process. The results also showed that patients in the two groups were homogeneous, hence having no significant difference in terms of mean age, gender, having a companion, and transfer characteristics (including risk rate, origin ward, transfer vehicle, transfer shift, receiving vasopressor drug and positive inotrope). Nonetheless, they were not homogeneous with respect to items such as medical diagnosis, transfer reason, destination ward, physician specialty, transfer duration, and transfer team members. This disparity could be related to the hospital examined and the services it provides. Thus, Khatam Hospital (control group) is the oldest and busiest medical center in the east and southeast of Iran which admits trauma patients, while Ali ebne Abi Taleb Hospital is a relatively new health center (about 15 years), which provides services in all specialized medical fields. The nurses in the two groups were homogeneous in terms of age, gender, level of education, work shift, experience in general and specialized wards, and attending the educational program on patient transfer, however, they differed with regard to marital status and employment status.

The results of the present study demonstrated that the quality of critical patient transfer in the control group hospital was not favorable in the majority of cases. Studies conducted in Iran and abroad, aimed at describing the status of patient transfers (Adib-HajBagheri et al. (7) and Habibzadeh et al. (22)), have suggested that most intrahospital transfers do not have a desirable quality, and, therefore, may compromise patient safety. Borimnejad et al. (23), reported that intrahospital transfer of high-risk neonates in Tehran is far from international standards. In fact, they mentioned that the status of neonate oxygenation was not controlled in more than half of transfer cases; furthermore, most transfers lacked sufficient equipment and medications. As a result, the researchers recommended that nurses responsible for moving these patients receive appropriate training in transferring highrisk neonates (23). The results of foreign studies also suggest the unfavorable quality of transferring high-risk patients $(6,11,24)$. Given that neither intrahospital nor interhospital transfer is trained during undergraduate nursing education in Iran and considering the gap in the knowledge of nurses regarding relevant standards, this suggestion is understandable. Hence the need for devising and implementing educational interventions for nursing students and practitioners.

The results of this study proposed a greater transfer quality in the intervention group, compared to the control group, after providing safe transfer instructions and encouraging nurses to make use of the transfer checklist. This finding corroborates that training nurses to use transfer safety checklists has a positive effect on their performance, such that one month after the training, the nurses began abiding by the standards of patient transfer. Investigating the effect of face-to-face education and using safe transfer checklists, Habibzadeh et al. (22, 25), reported that the quality of transferring ICU patients improved after training nurses and requiring them to use the checklist.

In line with our research, foreign studies have attained similar results. For instance, Stevenson et al. (10), stated that training can significantly increase nurses' knowledge and, consequently, enhance patient safety. Implementing an interdisciplinary prevention program, which involved all members of the treatment team in transferring critical patients, Berube et al. (26), reported an improvement in the transfer quality, such that the incidence of adverse events during transfer decreased. The authors recommended adopting this intervention as a care standard for avoiding unfavorable incidents when transferring critical patients. In another study, the results supported that educating nurses and using performance checklists could significantly prevent unexpected incidents during the intrahospital transfer of emergency patients (8). Silva and Amante (16), showed that most adverse events occurring during transferring patients were due to inefficient equipment and devices. In this regard, hospitals can ensure patient safety during patient transfers by requiring nurses to use standard checklists (16). In a study designed to provide a checklist for improving intrahospital transfers, Brunsveld-Reinders et al. (27), examined the incidence of adverse events during patient transfers over a 36-month 


\begin{tabular}{|c|c|c|c|}
\hline Transfer characteristics & Control Group & Intervention Group & Result \\
\hline Medical diagnosis & & & $\mathrm{P}=0.001^{\mathrm{b}}$ \\
\hline Internal neurology & $5(7.7)$ & $7(10.8)$ & \\
\hline Heart and lung & $4(6.2)$ & $21(32.3)$ & \\
\hline Multiple trauma & $43(66.2)$ & $7(10.8)$ & \\
\hline Gastrointestinal diseases & $2(3.1)$ & $9(13.8)$ & \\
\hline Reduced consciousness & $6(9.2)$ & $8(12.3)$ & \\
\hline Other diagnoses & $5(7.7)$ & $13(20)$ & \\
\hline Specialty of treating physician & & & $\mathrm{P}=0.001^{\mathrm{b}}$ \\
\hline Emergency medicine specialist & $15(23.1)$ & $5(7.7)$ & \\
\hline Neurosurgery & $22(33.8)$ & $3(4.6)$ & \\
\hline Internal neurology & $6(9.2)$ & $11(16.9)$ & \\
\hline General surgery & $5(7.7)$ & $20(30.8)$ & \\
\hline Internal specialty and subspecialty & $3(4.6)$ & $16(24.6)$ & \\
\hline Others & $14(21.5)$ & $10(15.4)$ & \\
\hline Transfer time & & & $0.672^{\mathrm{b}}$ \\
\hline Morning shift & $36(55.4)$ & $31(47.7)$ & \\
\hline Evening shift & $21(32.3)$ & $24(36.9)$ & \\
\hline Night shift & $8(12.3)$ & $10(15.4)$ & \\
\hline Transfer reason & & & $\mathrm{P}=0.001^{\mathrm{b}}$ \\
\hline Head/chest/abdominal CT scan & $28(43.1)$ & $14(21.5)$ & \\
\hline Hospitalization & $19(29.2)$ & $30(46.2)$ & \\
\hline Radiography & $12(18.5)$ & $1(1.5)$ & \\
\hline Surgery & $4(6.2)$ & $8(12.3)$ & \\
\hline Other reasons & $2(3.1)$ & $12(18.5)$ & \\
\hline Origin ward & & & $0.482^{\mathrm{b}}$ \\
\hline ICU & $33(50.8)$ & $37(56.9)$ & \\
\hline Emergency & $32(49.2)$ & $28(43.1)$ & \\
\hline Destination ward & & & $\mathrm{P}=0.001^{\mathrm{b}}$ \\
\hline Special units (ICU, CCU, Dialysis) & $19(29.2)$ & $34(52.3)$ & \\
\hline Radiology and ultrasonography & $9(13.8)$ & $2(3.1)$ & \\
\hline CT scan & $30(46.2)$ & $14(21.5)$ & \\
\hline Internal medicine/surgery & $2(3.1)$ & $4(6.2)$ & \\
\hline Operating suite & $5(7.7)$ & $11(16.9)$ & \\
\hline Transfer vehicle & & & $0.858^{\mathrm{b}}$ \\
\hline Hospital bed & $26(40)$ & $27(41.5)$ & \\
\hline Stretcher & $39(60)$ & $38(58.5)$ & \\
\hline
\end{tabular}

${ }^{\mathrm{a}}$ Values are expressed as No. (\%).

${ }^{\mathrm{b}}$ Chi-square.

period and found that more than half of the unexpected incidents take place during the transfer process. Transfer team members mentioned forgetfulness and emergency nature of the patient as the most important reasons for not using a checklist. Eventually, it was concluded that physicians and nurses could feel more confident if they received more practical training. Nurses also acknowledged that, besides its usability, transfer checklist serves as a complementary role and reduces the likelihood of forgetting important items (27). Consistent with the present study, Ligtenberg et al. (28) concluded that patient transfer is enhanced by accurate usage of standard checklists and guidelines and establishing better communication between referring and receiving hospitals prior to the transfer pro- 
Table 2. Comparing the Quality of Transferring Critical Patients in the Control and Intervention Groups ${ }^{\mathrm{a}}$

\begin{tabular}{lccc}
\hline Group & Intervention & Control & PValue \\
\hline Transfer quality & $16.52 \pm 2.08$ & $11.21 \pm 2.84$ & $0.001^{\mathrm{b}}$ \\
\hline
\end{tabular}

${ }^{\mathrm{a}}$ Values are expressed as mean $\pm \mathrm{SD}$.

${ }^{\mathrm{b}}$ Independent $t$-test.

cess (28). Although studies in this area suggest that the quality of transferring critical patients has ameliorated over the past 40 years, it is increasingly emphasized that critical patients need to be transferred by specialist teams (29).

\subsection{Conclusions}

The results of the present study propose that the quality of intrahospital transfer of critical patients is not satisfactory and does not comply with the safety standards of patient transfer. Therefore, appropriate workshops should be designed and implemented for the target group (ICU and Emergency nurses). Also, in order to increase the safety and quality of patient transfer, it is imperative to incorporate standards and guidelines of patient transfer in the curriculum of nursing students and in-service training programs of practicing nurses.

The major limitation of this study concerns the adoption of direct observation method to complete questionnaires. This could have influenced nurses' behavior [in abiding by safety measures of patient transfer], which was out of the control of the researcher.

\section{Acknowledgments}

The authors are grateful to the members of the Research Council of the School of Nursing and Midwifery, the Research Council of Zahedan University of Medical Sciences, the Ethics Committee of the University, and all nurses and patients as well as their companions who contributed to this study.

\section{Footnotes}

Authors' Contribution: Zahra Pishkar Mofrad: study design, research supervision, scientific revision, and final approval; Gholam Hosein Sargazi: statistical analysis; Leila Safabakhsh: scientific advisor; and Sadegh Akrami: sampling and manuscript preparation.

Clinical Trial Registration Code: The code is IRCT20180614040096N1.

Conflict of Interests: This study was funded by Zahedan University of Medical Sciences.
Ethical Approval: The code is IRCT20180614040096N1. Funding/Support: This study was funded by Zahedan University of Medical Sciences.

Informed Consent: All participants were informed consent.

\section{References}

1. Day D. Keeping patients safe during intrahospital transport. Crit Care Nurse. 2010;30(4):18-32. quiz 33. doi: 10.4037/ccn2010446. [PubMed: 20436033].

2. McCunn M, Mirvis S, Reynolds N, Cottingham C. Physician utilization of a portable computed tomography scanner in the intensive care unit. Crit Care Med. 2000;28(12):3808-13. doi: 10.1097/00003246200012000-00008. [PubMed: 11153618].

3. Porter JM, Ivatury RR, Kavarana M, Verrier R. The surgical intensive care unit as a cost-efficient substitute for an operating room at a level I trauma center. Am Surg. 1999;65(4):328-30. [PubMed: 10190356].

4. Warren J, Fromm RJ, Orr RA, Rotello LC, Horst HM; American College of Critical Care Medicine. Guidelines for the inter- and intrahospital transport of critically ill patients. Crit Care Med.2004;32(1):256-62. doi: 10.1097/01.CCM.0000104917.39204.0A. [PubMed: 14707589].

5. Ferdinande P. Recommendations for intra-hospital transport of the severely head injured patient. Working Group on Neurosurgical Intensive Care of the European Society of Intensive Care Medicine. Intensive Care Med. 1999;25(12):1441-3. doi: 10.1007/s001340051096. [PubMed: 10660856].

6. Lahner D, Nikolic A, Marhofer P, Koinig H, Germann P, Weinstabl C, et al. Incidence of complications in intrahospital transport of critically ill patients-experience in an Austrian University Hospital. Wien Klin Wochenschr. 2007;119(13-14):412-6. doi: 10.1007/s00508-007-08134. [PubMed: 17671822].

7. Adib-HajBagheri M, Afazel M, Safari Z. Quality of intra-hospital patient transports in Kashans' Beheshti Hospital. Rostamineh. 2012;3(4):58-65.

8. Choi HK, Shin SD, Ro YS, Kim DK, Shin SH, Kwak YH. A beforeand after-intervention trial for reducing unexpected events during the intrahospital transport of emergency patients. Am J Emerg Med. 2012;30(8):1433-40. doi: 10.1016/j.ajem.2011.10.027. [PubMed: 22205013].

9. Mohammadi G, Ebrahimian AA, Mahmoudi H. Evaluating the knowledge of intensive care unit nursing staffs. Iran J Critic Care Nurs. 2009;2(1):41-6.

10. Stevenson A, Fiddler C, Craig M, Gray A. Emergency Department Organisation of Critical Care Transfers in the UK. Emerg Med J. 2005;22(11):795-8. doi: 10.1136/emj.2004.017822. [PubMed: 16244338]. [PubMed Central: PMC1726606].

11. Beckmann U, Gillies DM, Berenholtz SM, Wu AW, Pronovost P. Incidents relating to the intra-hospital transfer of critically ill patients. An analysis of the reports submitted to the Australian Incident Monitoring study in Intensive Care. Intensive Care Med. 2004;30(8):1579-85. doi: 10.1007/s00134-004-2177-9. [PubMed: 14991102].

12. Hales B, Terblanche M, Fowler R, Sibbald W. Development of medical checklists for improved quality of patient care. Int J Qual Health Care. 2008;20(1):22-30. doi: 10.1093/intqhc/mzm062. [PubMed: 18073269].

13. Walsh TS, Dodds S, McArdle F. Evaluation of simple criteria to predict successful weaning from mechanical ventilation in intensive care patients. Br J Anaesth. 2004;92(6):793-9. doi: 10.1093/bja/aeh139. [PubMed: 15121724].

14. Winters BD, Gurses AP, Lehmann H, Sexton JB, Rampersad CJ, Pronovost PJ. Clinical review: Checklists - translating evidence into practice. Crit Care. 2009;13(6):210. doi: 10.1186/cc7792. [PubMed: 20064195]. [PubMed Central: PMC2811937]. 
15. Schroter D. Sustainability evaluation checklist. Michigan: Western Michigan University; 2010

16. Silva R, Amante LN. Checklist for the intrahospital transport of patients admitted to the Intensive Care Unit. Texto Contexto Enfermagem. 2015;24(2):539-47. doi: 10.1590/0104-07072015001772014.

17. Farnoosh L, Hossein-Nejad H, Beigmohammadi MT, Seyed-HosseiniDavarani SH. Preparation and implementation of intrahospital transfer protocol for Emergency Department Patients to decrease unexpected events. Adv J Emerg Med. 2018;2(3). e29. doi: 10.22114/AJEM.v0i0.50. [PubMed: 31172092]. [PubMed Central: PMC6549197].

18. Zuchelo LT, Chiavone PA. Intrahospital transport of patients on invasive ventilation: Cardiorespiratory repercussions and adverse events. J Bras Pneumol. 2009;35(4):367-74. doi: 10.1590/s180637132009000400011. [PubMed: 19466275].

19. Stearley HE. Patients' outcomes: Intrahospital transportation and monitoring of critically ill patients by a specially trained ICU nursing staff. Am J Crit Care. 1998;7(4):282-7. [PubMed: 9656042].

20. Habibzadeh F, Aliha JM, Imanipour M, Mehran A. The effect of interactive workshop on the quality of intra-hospital patients' transfer: A Quasi-Experimental study. Nurs Pract Today. 2017;4(2):79-86.

21. Markakis C, Dalezios M, Chatzicostas C, Chalkiadaki A, Politi K Agouridakis P. Evaluation of a risk score for interhospital transport of critically ill patients. Emerg Med J. 2006;23(4):313-7. doi 10.1136/emj.2005.026435. [PubMed: 16549583]. [PubMed Central: PMC2579512].

22. Habibzadeh F, Imanipour M, Mohammad Aliha J, Mehran A. Effect of applying checklist on quality of intra-hospital transport of intensive care patients. Iran J Cardiovasc Nurs. 2014;3(3):30-7.
23. Borimnejad L, Ansari K, Tatarpour P, Haghani H. Studying high risk newborn intra-hospital transport in Neonatal Intensive Care Unit (NICU). Iran J Crit Care Nurs. 2013;6(3):199-206.

24. Mendes KDS, Silveira RC, Galvão CM. [Integrative literature review: a research method to incorporate evidence in health care and nursing]. Texto Contexto Enfermagem. 2008;17(4):758-64. Brazilian. doi: 10.1590/s0104-07072008000400018.

25. Habibzadeh F, Aliha JM, Imanipour M, Mehran A. Examining the effect of interactive workshop on nurses' performance regarding safe patient transfer. Nurs Pract Today. 2017;4(2).

26. Berube M, Bernard F, Marion H, Parent J, Thibault M, Williamson DR, et al. Impact of a preventive programme on the occurrence of incidents during the transport of critically ill patients. Intensive Crit Care Nurs. 2013;29(1):9-19. doi: 10.1016/j.iccn.2012.07.001. [PubMed: 22921453].

27. Brunsveld-Reinders AH, Arbous MS, Kuiper SG, de Jonge E. A comprehensive method to develop a checklist to increase safety of intrahospital transport of critically ill patients. Crit Care. 2015;19:214. doi: 10.1186/s13054-015-0938-1. [PubMed: 25947327]. [PubMed Central: PMC4438434].

28. Ligtenberg JJ, Arnold LG, Stienstra Y, van der Werf TS, Meertens JH, Tulleken JE, et al. Quality of interhospital transport of critically ill patients: A prospective audit. Crit Care. 2005;9(4):R446-51. doi: 10.1186/cc3749. [PubMed:16137359]. [PubMed Central: PMC1269465].

29. Droogh JM, Smit M, Absalom AR, Ligtenberg JJ, Zijlstra JG. Transferring the critically ill patient: Are we there yet? Crit Care. 2015;19:62. doi: 10.1186/s13054-015-0749-4. [PubMed: 25887575]. [PubMed Central: PMC4335540]. 